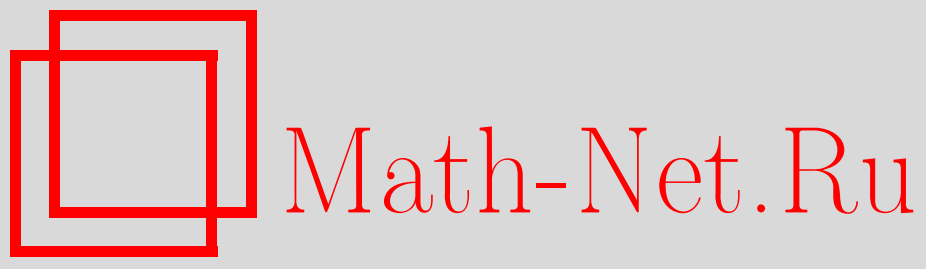

В. И. Арнольд, Динамика Ферма, арифметика матриц, конечная окружность и конечная плоскость Лобачевского, Функи. анализ и его прил., 2004, том 38, выпуск 1, 1-15

DOI: https://doi.org/10.4213/faa92

Использование Общероссийского математического портала MathNet.Ru подразумевает, что вы прочитали и согласны с пользовательским соглашением

http://www . mathnet.ru/rus/agreement

Параметры загрузки:

IP: 54.162 .27 .143

26 апреля 2023 г., 14:27:49

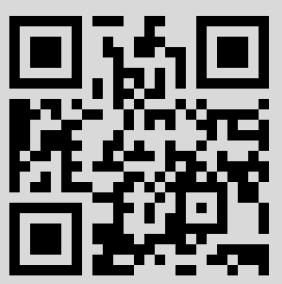


Функииональньй анализ и его приложения

2004, т. 38, вып. 1, с. 1-15

УДК 51

\title{
Динамика Ферма, арифметика матриц, конечная окружность и конечная плоскость Лобачевского*
}

\author{
(с) 2004. В. И. Арнольд
}

Динамическая система Ферма сопоставляет в качестве своей траектории каждому вычету по модулю $n$ геометрическую прогрессию его степеней. В настоящей статье исследуется матричное обобщение этой динамики, прежде всего в случае целочисленных матриц второго порядка с определителем единица, составленных из вычетов по простому модулю (хотя нетривиальные факты получаются и для матриц любого порядка).

Наряду с информацией о топологии операции возведения матриц из вычетов в квадрат и об описывающих эту операцию графах и деревьях мы получим, например, матричные аналоги малой теоремы Ферма и теории первообразных вычетов, а также укажем на связь всего этого предмета с геометриями Лобачевского и де Ситтера над конечными полями, неожиданно появляющимися при исследовании уравнений $A^{m}=1$ в группах матриц: аналитические неравенства заменяются в теории чисел условием квадратичности вычета.

Мы также изучим ниже коммутатор операций суммирования и возведения в фиксированную степень элементов кольца.

\section{§1. Динамика следов}

Пусть $A$ - целочисленная матрица порядка $r, \operatorname{tr} A-$ ее след, $p$ - простое число. Рассматривая следы степеней этой матрицы, мы приходим к следующему обобщению малой теоремы Ферма.

Теорема 1. Имеет место сравнение по модулю $p, \operatorname{tr}\left(A^{p}\right) \equiv(\operatorname{tr} A)^{p}$.

Более того, разность правой и левой частей представляет собой многочлен (от коэффичиентов характеристического полинома матрицы А) с иельми коэфбичиентами, делящимися на р:

$$
(\operatorname{tr} A)^{p}-\operatorname{tr}\left(A^{p}\right)=p F_{p}\left(\sigma_{1}, \ldots, \sigma_{r}\right), \quad F_{p} \in \mathbb{Z}[\sigma],
$$

$\sigma=\left(\sigma_{1}, \ldots, \sigma_{r}\right)$, где $\sigma_{k}-k$-я основная симметрическая функция от корней характеристического уравнения:

$$
\sigma_{1}=\operatorname{tr} A=\sum \lambda_{j}, \sigma_{2}=\sum \lambda_{j} \lambda_{m}, \ldots, \quad \sigma_{r}=\operatorname{det} A=\prod \lambda_{j}=1 .
$$

Теорема 1 в сущности эквивалентна аналогичному ей утверждению о многочленах:

Tеорема 1'. Имеет место тождество

$$
\left(\lambda_{1}+\cdots+\lambda_{r}\right)^{p}-\left(\lambda_{1}^{p}+\cdots+\lambda_{r}^{p}\right)=p F_{p}(\sigma(\lambda)), \quad F_{p} \in \mathbb{Z}\left[\sigma_{1}, \ldots, \sigma_{p}\right]
$$

*Частично поддержано грантом РФФИ 02-01-00655. 
(в котором взвешенно-однородный многочлен Нъютона-Жирара $F_{p}$ взвешенной степени $p$ при $\operatorname{deg} \sigma_{k}=k$ не зависит вдобавок от числа аргументов $r$ ).

ПримеР 1. $\left(\lambda_{1}+\lambda_{2}\right)^{3}-\left(\lambda_{1}^{3}+\lambda_{2}^{3}\right)=3\left(\lambda_{1}+\lambda_{2}\right) \lambda_{1} \lambda_{2}$. Наши теоремы обобщают это соответствующее $r=2, p=3$ тождество (и другие аналогичные теоремы о делимости элементов треугольника Паскаля) на случай бо́льших значений $r$ и $p$.

ПримеР 2. Для единичной матрицы $A$ (или для набора $\lambda_{j} \equiv 1$ ) наши теоремы принимают вид сравнения

$$
r^{p} \equiv r \quad(\bmod p),
$$

т. е. вид малой теоремы Ферма. В этом смысле теорема 1 является матричным аналогом малой теоремы Ферма.

ПримеР 3. Для $p=2,3,5$ и 7 явные формулы для многочленов НьютонаЖирара $F_{p}$ теорем 1 и $1^{\prime}$ оказываются удивительно короткими (см. $\left.[4,5]\right)$ :

$$
\begin{aligned}
\left(\lambda_{1}+\cdots+\lambda_{r}\right)^{2}-\left(\lambda_{1}^{2}+\cdots+\lambda_{r}^{2}\right)=2 \sigma_{2}, \\
\left(\lambda_{1}+\cdots+\lambda_{r}\right)^{3}-\left(\lambda_{1}^{3}+\cdots+\lambda_{r}^{3}\right)=3\left(\sigma_{1} \sigma_{2}-\sigma_{3}\right), \\
\left(\lambda_{1}+\cdots+\lambda_{r}\right)^{5}-\left(\lambda_{1}^{5}+\cdots+\lambda_{r}^{5}\right)=5\left(\sigma_{1}^{3} \sigma_{2}-\sigma_{1}^{2} \sigma_{3}+\sigma_{1} \sigma_{4}-\sigma_{1} \sigma_{2}^{2}+\sigma_{2} \sigma_{3}-\sigma_{5}\right), \\
\left(\lambda_{1}+\cdots+\lambda_{r}\right)^{7}-\left(\lambda_{1}^{7}+\cdots+\lambda_{r}^{7}\right)=7\left(\sigma_{1}^{5} \sigma_{2}-\sigma_{1}^{4} \sigma_{3}+\sigma_{1}^{3} \sigma_{4}-2 \sigma_{1}^{3} \sigma_{2}^{2}-\sigma_{1}^{2} \sigma_{5}\right. \\
+3 \sigma_{1}^{2} \sigma_{2} \sigma_{3}+\sigma_{1} \sigma_{6}-2 \sigma_{1} \sigma_{2} \sigma_{4}-\sigma_{1} \sigma_{3}^{2} \\
\left.+\sigma_{1} \sigma_{2}^{3}-\sigma_{7}+\sigma_{2} \sigma_{5}+\sigma_{3} \sigma_{4}-\sigma_{2}^{2} \sigma_{3}\right) .
\end{aligned}
$$

В этих формулах неожиданно много коэффициентов многочлена $F_{p}$ оказываются равными +1 и -1 (причем числа положительных и отрицательных коэффициентов для $F_{p>2}$ одинаковы при $p \leqslant 7$ ), и все коэффициенты малы.

Эти коэффициенты можно считать функциями от диаграмм Юнга разбиений $p=\sum a_{k}$, соответствующих одночленам $\prod \sigma_{a_{k}}$. Коэффициент при «одночлене» $\prod\left(\sigma_{s}^{m_{s}}\right)$ в многочлене $\sum \lambda_{j}^{n}$ равен произведению

$$
\operatorname{Coeff}\left[\prod\left(\sigma_{s}^{m_{s}}\right)\right]=(-1)^{n-m}(n / m) C\left(\left\{m_{s}\right\}\right),
$$

где число $n=\sum s m_{s}-$ квазиоднородная степень одночлена, т. е. его степень по переменным $\lambda$, число $m=\sum m_{s}$ - число перемножаемых базисных симметрических функций, а $C$ - мультиномиальный коэффициент, вычисляемый по формуле

$$
C\left(\left\{m_{s}\right\}\right)=m ! /\left(\prod\left(m_{s} !\right)\right) .
$$

Коэффициент $C$ равен числу $m$-буквенных слов с $m_{s}$ вхождениями буквы $s$. Асимптотика его логарифма (при $m_{s} \rightarrow \infty, m_{s} / m \rightarrow p_{s}$ ) порождает энтропию $\sum\left(p_{s} \log p_{s}\right)$ в теории информации:

$$
\lim \left(\log C\left(\left\{m_{s}\right\}\right) / m\right)=\sum\left(p_{s} \log p_{s}\right) .
$$

Эти коэффициенты $C$ образуют многомерный аналог треугольника Паскаля.

Степень $n$ в предыдущих формулах - число не обязательно простое, так что они пригодны для перехода от малой теоремы Ферма к обобщающей ее теореме Эйлера, $a^{n} \equiv a^{n-\varphi(n)}(\bmod n)$. 
ПРИМЕРЫ.

$$
\begin{gathered}
\text { Coeff }\left[\sigma_{1}^{2} \sigma_{2}^{2} \sigma_{3}\right]=54 \quad(\text { где } n=9, m=5), \\
\text { Coeff }\left[\sigma_{1}^{a} \sigma_{2} \sigma_{3}\right]=-n(n-4) \quad(\text { где } n=a+5, m=a+2), \\
\operatorname{Coeff}\left[\sigma_{u}^{a} \sigma_{v}^{b}\right]= \pm(n /(a+b)) C_{a+b}^{a} \quad(\text { где } u \neq v) .
\end{gathered}
$$

Доказательство приведенной формулы $(*)$ для Coeff получается из рекуррентной формулы Ньютона-Кирара для $s_{n}=\sum\left(\lambda_{j}^{n}\right)$, вытекающей из уравнения теоремы Виета $\lambda^{n}=\sigma_{1} \lambda^{n-1}-\sigma_{2} \lambda^{n-2}+\ldots$ :

$$
s_{n}=\sum(-1)^{q+1} \sigma_{q} s_{n-q} \quad\left(\text { где } s_{0}=n\right) .
$$

Указанные значения Coeff удовлетворяют этим соотношениям вследствие следующего обобщения определяющего свойства треугольника Паскаля (для чисел $C(a, b)=\operatorname{Coeff}\left[\sigma_{u}^{a} \sigma_{v}^{b}\right]$ и аналогичных чисел $C\left(a_{1}, \ldots, a_{r}\right)$ с попарно различными весами $\left.\left(u_{1}, \ldots, u_{r}\right)\right)$ :

$$
C\left(a_{1}, \ldots, a_{r}\right)=\sum(-1)^{u_{s}+1} C\left(a_{1}, \ldots, a_{s-1}, a_{s}-1, a_{s+1}, \ldots, a_{r}\right) .
$$

Это тождество $(* *)$ выводится из рекуррентного соотношения Ньютона-Жираpa, приведенного выше, заменой входящих в последнее величин $s_{k}$ их выражениями $s_{k}=\sum(C \Pi)$ (и приравниванием коэффициентов при одинаковых произведениях базисных симметрических функций).

Рекуррентное соотношение $(* *)$ для коэффициентов $C$ влечет за собой выражение $(*)$ этих коэффициентов в терминах мультиномальных коэффициентов.

Действительно, при замене коэффициентов $C$ в формуле (**) их выражениями $(*)$ мы получим для мультиномиальных коэффициентов правильное тождество (типа соотношения Паскаля для биномиальных коэффициентов), в справедливости которого легко убедиться либо считая, как Паскаль, пути (в многомерном пространстве), либо прямо складывая выражения мультиномиальных коэффициентов через факториалы.

Итак, числа $(*)$ удовлетворяют тождеству $(* *)$.

Из этого вытекает справедливость формулы $(*)$, так как рекуррентные соотношения $(* *)$ типа Паскаля, очевидно, имеют только одно решение, а числа (*) этим рекуррентным соотношениям удовлетворяют.

ДокАЗАТЕЛЬСТво теорем 1 и $1^{\prime}$. Произведение $p$ одинаковых сомножителей $\lambda_{1}+\cdots+\lambda_{r}$ является суммой $r^{p}$ одночленов степени $p$, каждый из которых имеет вид произведения степеней различных аргументов, $\mu^{a}=\mu_{1}^{a_{1}} \cdots \mu_{s}^{a_{s}}$, где $\mu_{k}=\lambda_{j}(k)$, причем $\sum a_{k}=p, s \leqslant p$.

Выписанный одночлен $\mu^{a}$ повторяется в сумме несколько раз, так как, например, $\mu_{1}^{a_{1}}$ может быть вкладом какого-то набора из $a_{1}$ сомножителей, из числа $p$ сомножителей, $\mu_{2}^{a_{2}}$ - вкладом какого-то (не пересекающегося с первым) другого набора из $a_{2}$ сомножителей, и так далее. Но выбор этих непересекающихся поднаборов произволен.

Поэтому общее число повторений выписанного одночлена $\mu^{a}$ в нашей сумме $r^{p}$ слагаемых дается произведением чисел сочетаний, считающих последовательные выборы отдельных поднаборов,

$$
N(a)=C_{p_{1}}^{a_{1}} C_{p_{2}}^{a_{2}} \cdots C_{p_{s}}^{a_{s}}
$$


(где $\left.p_{1}=a_{1}+\cdots+a_{s}=p, p_{2}=a_{2}+\cdots+a_{s}, \ldots, p_{s}=a_{s}\right)$. Действительно, для выбора первого набора имеется $C_{p_{1}}^{a_{1}}$ возможностей. Элементы второго поднабо$\mathrm{pa}$, из $a_{2}$ сомножителей, равных $\mu_{2}$, выбираются из $p_{2}=p-a_{1}$ не входящих в первый поднабор сомножителей, и так далее.

Заметим теперь, что число сочетаний

$$
C_{p_{1}}^{a_{1}}=\frac{p(p-1) \cdots\left(p-a_{1}+1\right)}{a_{1} !}
$$

делится на $p$, так как число $p$ простое, а число $a_{1}$ ! не имеет простого делителя $p$ при $a_{1}<p$.

Следовательно, выписанное выше произведение целых сомножителей, $N$, делится на $p$.

Аналогичный исследованному одночлену $\mu^{a}$ одночлен $\tilde{\mu}^{a}$, получающийся из него при замене попарно различных аргументов $\mu_{k}$ попарно различными аргументами $\tilde{\mu}_{k}$ (из тех же $\lambda_{j}$ ), входит в сумму с такой же кратностью, как исследованный одночлен $\mu^{a}$ (например, $\lambda_{1}^{2} \lambda_{2}^{2} \lambda_{3}$ входит столько же раз, сколько $\left.\lambda_{1}^{2} \lambda_{4}^{2} \lambda_{2}\right)$.

Поэтому вся наша сумма представляет собой сумму симметрических многочленов от $\lambda$ с делящимися на $p$ коэффициентами $N(a)$ (соответствующих разным диаграммам Юнга $a$ площади $p=\sum a_{k}$ ). Выражая эти многочлены через элементарные, мы и получим представление их суммы в указанном в теореме $1^{\prime}$ виде (а тем самым докажем и теорему 1 , вытекающую из теоремы $1^{\prime}$ ).

ДоБАВЛЕНИЕ. Число сочетаний $C_{m}^{k}$, где $m$ и $k$ взаимно просты, делится на $m$, причем число сочетаний $C_{m-1}^{k-1}$ делится на $k$.

Первое доказывается при отождествлении числа сочетаний с числом $k$-элементных подмножеств $X$ в $\mathbb{Z}_{m}$. Сдвиги $x \mapsto x+a($ где $a=1, \ldots, m)$ переводят $X$ в $m$ различных $k$-элементных подмножеств. Ибо иначе нашелся бы сдвиг, переводящий $X$ в себя. Его период $T$ должен быть делителем и $m$ и $k$, вопреки их взаимной простоте (так как $m$-кратное повторение этого сдвига - тождественное преобразование, а $X$ разбивается на $T$-элементные орбиты этого сдвига). Второе утверждение следует из первого.

Так же доказывается делимость на $m$ мультиномиального коэффициента $m ! /\left(k_{1} !\right) \cdots\left(k_{s} !\right), m=\sum k_{i}$, если одно из чисел $k_{i}$ взаимно просто с $m$.

ПримеР. Для матриц $A$ порядка $n=2$ с определителем 1 теорему 1 можно переписать следующим образом. Обозначим след матрицы $A^{m}$ через $t_{m}=$ $\operatorname{tr}\left(A^{m}\right)$.

УТВЕРЖДЕНИЕ. След $t_{m}-$ многочлен $с$ иелыми коэффициентами от $t=$ $t_{1}=\operatorname{tr} A$.

Действительно, матрица удовлетворяет своему характеристическому уравнению,

$$
A^{2}=A t-1, \quad A^{m+1}=A^{m} t-A^{m-1} .
$$

Следовательно, если $\operatorname{tr}\left(A^{m}\right)=t_{m}(t)$, то

$$
t_{m+1}(t)=t t_{m}(t)-t_{m-1}(t),
$$


так что из начальных условий $t_{0}(t)=\operatorname{tr}(1)=2, t_{1}(t)=t$ мы находим последовательность многочленов от одной переменной $t$ :

$$
\begin{gathered}
\quad t_{0}=2, \quad t_{1}=t, \quad t_{2}=t^{2}-2, \quad t_{3}=t^{3}-3 t, \quad t_{4}=t^{4}-4 t^{2}+2, \\
t_{5}=t^{5}-5 t^{3}+5 t, \quad t_{6}=t^{6}-6 t^{4}+9 t^{2}-2, \quad t_{7}=t^{7}-7 t^{5}+14 t^{3}-7 t .
\end{gathered}
$$

Общий член этой последовательности имеет вид

$$
t_{m}(t)=\sum A_{k, l} t^{k}, \quad \text { где } k+2 l-2=m, 0 \leqslant k \leqslant m
$$

(разумеется, суммирование по $k$ той же четности, что $m$ ), где коэффициенты

$$
A_{k, l}=\frac{l(l+1) \cdots(l+k-2)(2 l+k-2)}{k !}(-1)^{l-1}
$$

образуют треугольник типа Паскаля. Начальная $(k=0,1, \ldots, 4)$ часть этого треугольника, без знакового множителя $(-1)^{l-1}$, имеет следующий вид:

$$
\begin{aligned}
& \begin{array}{llllllllll}
6 & 1 & 0 & 0 & 0 & 1 & 6 & 20 & 50 & 105
\end{array} \\
& \begin{array}{llllllllll}
-5 & -1 & 0 & 0 & 1 & 5 & 14 & 30 & 55 & 91
\end{array} \\
& \begin{array}{lllllllll}
4 & 1 & 0 & 1 & 4 & 9 & 16 & 25 & 36
\end{array} \\
& \begin{array}{lllllllll}
-3 & -1 & 1 & 3 & 5 & 7 & 9 & 11 & 13
\end{array} \\
& \begin{array}{llllllll}
2 & 2 & 2 & 2 & 2 & 2 & 2 & 2 .
\end{array}
\end{aligned}
$$

Строки этого «треугольника» задаются формулами

$$
\begin{gathered}
\pm A_{0, l}=2, \quad \pm A_{1, l}=2 l-1, \quad \pm A_{2, l}=l^{2}, \quad \pm A_{3, l}=\left(2 l^{3}+3 l^{2}+l\right) / 6, \\
\pm A_{4, l}=l(l+1)(l+2)(2 l+2) / 24 .
\end{gathered}
$$

Наши теоремы делимости относятся к вертикалям, расположенным ниже диагонали из единиц над простыми нечетными числами в ряду $\pm A_{1, l}$ : это вертикали

$$
\{3\} \text { для } p=3, \quad\{5,5\} \text { для } p=5, \quad\{7,14,7\} \text { для } p=7 \text {; }
$$

видно, что и дальше 55 делится на 11, а 91 на 13, как и должно быть по теореме 1 .

Наша последовательность доставляет также полезные многочлены $p_{m}$ и $q_{m}$, определяемые условиями $A^{m}=p_{m} \cdot A+q_{m} \cdot 1$, вытекающими из характеристического уравнения.

Для них характеристическое уравнение матрицы $A$ доставляет рекуррентные соотношения

$$
p_{m+1}=t p_{m}+q_{m}, \quad q_{m+1}=-p_{m}
$$

так что первые из этих полезных многочленов суть

$$
\begin{array}{ll}
p_{1}=1, & q_{1}=0, \\
p_{2}=t, & q_{2}=-1, \\
p_{3}=t^{2}-1, & q_{3}=-t, \\
p_{4}=t^{3}-2 t, & q_{4}=-t^{2}+1, \\
p_{5}=t^{4}-3 t^{2}+1, & q_{5}=-t^{3}+2 t, \\
p_{6}=t^{5}-4 t^{3}+3 t, & q_{6}=-t^{4}+3 t^{2}-1, \\
p_{7}=t^{6}-5 t^{4}+6 t^{2}-1, & q_{7}=-t^{5}+4 t^{3}-3 t .
\end{array}
$$


Многочлены $p_{m}, q_{m}$ и $t_{m}$ связаны очевидным соотношением аддитивности следов: $t_{m}=t p_{m}+2 q_{m}$.

\section{§2. Круговая группа $C_{p}$ на конечной плоскости}

Рассмотрим конечную окружность $C_{p}:\left\{x^{2}+y^{2}=1\right\}$ на конечной плоскости $\mathbb{Z}_{p}^{2}$, где $p$ - простое число. Хорошо известна

ЛЕмма 1. Число точек на окружности $C_{p}$ равно

$$
\left|C_{p}\right|= \begin{cases}p+1 & \text { nри } p=4 c-1 \\ p-1 & \text { при } p=4 c+1\end{cases}
$$

ПримеР. Для $p=5$ это 4 точки $(0, \pm 1)$ и $( \pm 1,0)$, а для $p=7$ - еще и 4 ТОЧКИ $( \pm 2, \pm 2)$.

ДокАЗАТЕЛЬСтво ЛЕммы 1. Параметризуем рациональную кривую $C_{p}$ «тангенсом $t$ половинного угла», т. е. угла наклона прямой, соединяющей точку кривой $(x, y)$ с ее точкой $(-1,0)$ :

$$
t=y /(1+x), \quad x=\left(1-t^{2}\right) /\left(1+t^{2}\right), \quad y=2 t /\left(1+t^{2}\right) .
$$

Число точек на окружности равно числу допустимых здесь значений проективного параметра $t=(1,2, \ldots, p ; \infty)$, при которых $1+t^{2} \neq 0$ (точка $(-1,0)$ соответствует значению параметра $t=\infty)$.

Чтобы решить уравнение асимптот, $t^{2}+1=0$, выразим $t$ через первообразный вычет $\rho$ по модулю $p$, положив $t=\rho^{\lambda}, 0<\lambda<p$. Поскольку $-1=\rho^{(p-1) / 2}$, для асимптотической точки $t$ показатель $\lambda$ должен удовлетворять сравнению

$$
2 \lambda=(p-1) / 2 \quad(\bmod (p-1)), \quad \text { т. е. } 4 \lambda=(p-1)(1+2 a), \quad a \in \mathbb{Z} .
$$

Поскольку нечетное число $2 a+1$ на 2 не делится, то $p-1=4 c$.

Итак, доказана

Лемма 2. Если р имеет вид $4 c-1$, то у уравнения $t^{2}+1=0$ решений в $\mathbb{Z}_{p}$ нет. Если же р имеет вид $4 c+1$, то указанный выше выбор $\lambda$ доставляет (единственные) два решения уравнения $t^{2}+1=0$ (одно из них соответствует $\lambda=c, a=0)$.

Вычитая из общего числа $p+1$ точек проективной оси $t$ указанное леммой 2 число не доставляющих точек окружности асимптотических значений параметра (это вычитаемое число асимптотических значений равно 0 или 2 соответственно для $p=-1$ и +1 по модулю 4), получаем лемму 1.

ОПРЕДЕЛЕНИЕ. Круговой группой $C_{p}$ называется множество всех матриц порядка 2 определителя 1 , составленных из вычетов по простому модулю $p$, имеющих вид матрицы умножения на комплексное число, т. е. вид

$$
A=\left(\begin{array}{cc}
x & y \\
-y & x
\end{array}\right) \text {. }
$$

Такая матрица имеет определитель 1 , если и только если $(x, y)$ - точка окружности $C_{p}$. Все такие матрицы образуют, очевидно, конечную коммутативную группу, которую мы будем также называть «окружностью $C_{p}$ ». Эта круговая группа является подгруппой группы унимодулярных матриц $G=\mathrm{SL}\left(2, \mathbb{Z}_{p}\right)$. 
ТЕОРема 2. Круговая группа $C_{p}$ является ииклической.

ДокАзАтЕльство. Как и каждая конечная коммутативная группа, круговая группа является прямым произведением циклических групп примарных порядков $q_{k}^{a_{k}}$, где $q_{k}$ - простые числа, вообще не обязательно разные. Докажем, что все эти простые числа, соответствующие разным примарным сомножителям, для круговой группы различны (например, что недопустимы произведения $\mathbb{Z}_{3}^{2}$ и $\left.\mathbb{Z}_{3} \mathbb{Z}_{9}\right)$.

ЛЕмма 3. Для любого натурального числа $m$ число решений $А$ уравнения $A^{m}=B$ на окружности $C_{p}$ не превосходит его степени $m>0$.

ДокАзАтельство. По формуле тангенса суммы параметр $t_{m}$ точки $A^{m}$ связан с параметром $t$ точки $A$ соотношением $t_{m}=f_{m}(t) / g_{m}(t)$, где многочлен $f_{m}$ имеет степень $m$ и многочлен $g_{m}$ степень $m-1$ при нечетном $m$ (степени $m-1$ и $m$ при четном $m$ ). Поэтому уравнение $A^{m}=B$ записывается в обоих случаях как алгебраическое уравнение степени $m$ относительно параметра $t$ : $t_{m} g_{m}(t)=f_{m}(t)$, а потому имеет не более $m$ корней. Лемма 3 доказана.

Но в группе $\mathbb{Z}_{q^{a}}$ имеется $q$ элементов степени $q$, так что в произведении $h$ примарных сомножителей $\mathbb{Z}_{q^{a}{ }_{k}}$ с общим $q$ число элементов степени $q$ равно $q^{h}>q$ при $h>1$.

Это доказывает, что $h_{k}=1$ и все числа $q_{k}$ в примарных сомножителях круговой группы различны. Значит, она является циклической, будучи прямым произведением циклических групп взаимно простых порядков. Теорема 2 тем самым доказана, и мы вычислили круговые группы:

$$
C_{p} \approx \begin{cases}\mathbb{Z}_{p+1} & \text { при } p=4 c-1 \\ \mathbb{Z}_{p-1} & \text { при } p=4 c+1\end{cases}
$$

Порядок круговой группы (с $p \neq 2$ ) всегда кратен 4 , как и должно быть вследствие 4-симметрии окружности.

ЗАмЕчАНиЕ. Группа $C_{2}$, исключенная в теореме, изоморфна $\mathbb{Z}_{2}$ и состоит из двух матриц перестановок координат, соответствующих точкам $(1,0)$ и $(0,1)$, составляющим $\mathbb{Z}_{2}$-окружность.

\section{§3. Динамика квадратов матриц порядка 2}

Рассмотрим операцию возведения в квадрат, $A \mapsto A^{2}$, матриц порядка 2 с определителем 1 , составленных из вычетов по простому модулю $p(A \in G=$ $\left.\mathrm{SL}\left(2, \mathbb{Z}_{p}\right)\right)$.

Итерируя эту операцию, мы строим по матрице $A$ последовательность матриц-квадратов из той же группы $G$ :

$$
A_{s}=A_{s-1}^{2}=A^{2^{s}}, \quad A_{0}=A, \quad A_{1}=A^{2}, \quad \ldots
$$

- траекторию «динамики квадратов».

ПРимеР. Для $p=7$ мы строим, например, цепочку квадратов

$$
A_{0}=\left(\begin{array}{ll}
2 & 1 \\
1 & 1
\end{array}\right), \quad A_{1}=\left(\begin{array}{ll}
5 & 3 \\
3 & 2
\end{array}\right), \quad A_{2}=\left(\begin{array}{cc}
-1 & 0 \\
0 & -1
\end{array}\right), \quad A_{3}=\left(\begin{array}{ll}
1 & 0 \\
0 & 1
\end{array}\right) .
$$

Длиной цепочки мы будем называть число $r$ шагов $(r=3$ возведения в квадрат в этом примере). 
Теорема 3. Цепочка квадратов длины $r$ (в которой $A_{r}=1, A_{s<r} \neq 1$ ) существует в группе $G=\mathrm{SL}\left(2, \mathbb{Z}_{p}\right)$, если и только если простое число р имеет вид $p=2^{r} c \pm 1$.

ПримеР. Для $p=7$ и 17 цепочки длины $r=3$ существуют, а для $p=5$ и 11 - нет, хотя есть цепочки длины $r=2$. Наибольшая длина цепочки равна $r$, если и только если $p=2^{r} c \pm 1$, где $c$ нечетно.

Доказательство теоремы состоит из ряда легких шагов.

ЛЕмма 1. Порлдок группъ $G=\mathrm{SL}\left(2, \mathbb{Z}_{p}\right)$ с простым $р$ равен $p\left(p^{2}-1\right)$.

ПримеР. $\left|\mathrm{SL}\left(2, \mathbb{Z}_{3}\right)\right|=24,\left|\mathrm{SL}\left(2, \mathbb{Z}_{5}\right)\right|=120,\left|\mathrm{SL}\left(2, \mathbb{Z}_{7}\right)\right|=336$.

ДокаЗАтЕЛЬСтво ЛЕммы 1. Для $A=\left(\begin{array}{ll}a & b \\ c & d\end{array}\right)$ из $G$ имеем $a d-b c=1$. Обозначим произведение $a d$ через $u \in \mathbb{Z}_{p}$. Тогда условие унимодулярности матрицы $A$ принимает вид

$$
a d=u, \quad b c=u-1 .
$$

Число способов упорядоченного разложения вычета $u$ на два множителя равно $p-1$, если $u \neq 0$, и $2 p-1$, если $u=0$. Подсчитывая отдельно вклады двух исключительных значений $u=0$ и $u=1$ и вклады остальных $p-2$ значений произведения $u$ (путем суммирования произведений чисел разложений обоих слагаемых $a d$ и $-b c$ ), мы получаем для порядка группы $G$ выражение

$$
\begin{aligned}
|G| & =2(p-1)(2 p-1)+(p-2)(p-1)^{2}=(p-1)\left[(4 p-2)+\left(p^{2}-3 p+2\right)\right] \\
& =(p-1)\left(p^{2}+p\right),
\end{aligned}
$$

что и доказывает лемму 1.

Цепочка квадратов $\left\{A_{s}\right\}$, заканчивающаяся членом $A_{r}=1$, определяет в группе $G$ циклическую подгруппу степеней $A_{0}^{k}$ элемента $A_{0}\left(k=1, \ldots, 2^{r}\right)$.

Лемма 2. Все эти $2^{r}$ элементов группы $G$ различны.

ДокАЗАТЕЛЬСтво. В противном случае было бы $A_{0}^{m}=1$ при некотором $m$ строго между 0 и $2^{r}$. Представим наибольший общий делитель $q$ чисел $m$ и $2^{r}$ в виде $q=2^{u}=U m+V 2^{r}$, где $u<r$. Тогда было бы $A_{0}^{q}=1$, т. е. $A_{u}=1$ при $u<r$, т. е. цепочка квадратов заканчивалась бы раньше $A_{r}$. Лемма 2 доказана.

Итак, группа $G$ порядка $p\left(p^{2}-1\right)$ содержит (циклическую) подгруппу порядка $2^{r}$. Из этого уже следует (при нечетном $p$ ), что либо $p+1$, либо $p-1$ делится на $2^{r-1}$. Мы докажем ниже делимость этого сомножителя на $2^{r}$.

ЛЕмма 3. Если в группе $G=\mathrm{SL}\left(2, \mathbb{Z}_{p}\right)$ имеется иепочка квадратов $\left\{A_{s}\right\}$ длины $r$ (так что $\left.A_{r}=1, A_{r-1} \neq 1\right)$, то такая чепочка квадратов имеется уже и в круговой подгруппе $C_{p}$ группъ $G$.

ДокАЗАтЕЛЬСтво. Рассмотрим последовательность следов матриц цепочки квадратов, $t_{s}=\operatorname{tr} A_{s}$. Из формулы возведения матриц в квадрат

$$
\left(\begin{array}{ll}
a & b \\
c & d
\end{array}\right)^{2}=\left(\begin{array}{ll}
a^{2}+b c & a b+b d \\
c a+d c & d^{2}+b c
\end{array}\right)=\left(\begin{array}{cc}
a t-1 & b t \\
c t & d t-1
\end{array}\right)
$$

где $t=\operatorname{tr} A=a+d$ (для матриц с определителем $a d-b c=1$ ), видно, что $t_{2}=\operatorname{tr} A^{2}=t^{2}-2, t_{s}=t_{s-1}^{2}-2(s=1, \ldots, r)$. Значит, цепочка следов квадратов, $\left\{t_{s}\right\}$, заканчивается членом $t_{r}=\operatorname{tr} 1=2$, перед которым стоит след $t_{r-1}$, для которого $t_{r-1}^{2}=4$. Следовательно, предпоследний след есть $t_{r-1}=2$ или -2 . 
Из формулы $(*)$ (при нечетном $p$ ) следует, что для матрицы $A_{r-1}$ элементы $b$ и $c$ равны 0 (поскольку $b t=c t=0$, ввиду $\left.A_{r}=1\right)$. Стало быть, эта матрица диагональная:

$$
A_{r-1}=\left(\begin{array}{ll}
a & 0 \\
0 & d
\end{array}\right), \quad a d=1, a+d= \pm 2 .
$$

Решая квадратное уравнение $a d=1$ относительно $a$, мы находим $a=d=1$ при $t=2$ и $a=d=-1$ при $t=-2$. И если $A_{r-1} \neq 1$, то предпоследним членом цепочки квадратов, заканчивающейся матрицей $A_{r}=1$, может быть только матрица $A_{r-1}=-1$ следа $t_{r-1}=-2$.

Чтобы закончить доказательство леммы 3, мы сначала исследуем предшествующий член цепочки, $A_{r-2}$ (подобно приведенному выше исследованию предпоследнего члена, $\left.A_{r-1}\right)$.

ОПРЕДЕЛЕНИЕ. Матрица из $G$ с квадратом -1 называется $G$-комплексной структурой.

ПРИмеР. Стандартная комплексная структура

$$
I=\left(\begin{array}{cc}
0 & 1 \\
-1 & 0
\end{array}\right) \in G=\operatorname{SL}\left(2, \mathbb{Z}_{p}\right)
$$

годится при любом $p$.

Лемма 4. Число $G$-комплексных структур для группы $G=\operatorname{SL}\left(2, \mathbb{Z}_{p}\right)$ равно $p(p \pm 1)$ при $p=4 c \pm 1$, и все они сопряжены стандартной структуре: $A_{r-2}=$ $g I g^{-1}$ для некоторого $g \in G$.

ДокАЗАтЕльство. След $t$ комплексной структуры удовлетворяет, согласно формуле $(*)$, соотношению $t^{2}-2=-2$, т. е. равен нулю. Обратно, если $t=\operatorname{tr} A=0$, то формула (*) показывает, что $A$ - комплексная структура $\left(A^{2}=-1\right)$.

Подсчитаем число матриц $A$ со следом нуль в группе $G$ :

$$
A=\left(\begin{array}{cc}
a & b \\
c & -a
\end{array}\right), \quad a^{2}+b c=-1
$$

Число упорядоченных разложений вычета $b c=u$ на множители $b$ и $c$ равно $p-1$, если $u \neq 0$, и равно $2 p-1$, если $u=0$. Число решений уравнения $a^{2}+1=0$ равно 0 при $p=4 c-1$ и равно 2 при $p=4 c+1$ (по лемме 2 из $\S 2$ ). Учитывая раздельно вклады особых значений $u=0$ и $u \neq 0$, мы получаем при $p=4 c-1$, где их нет,

$$
\#\left(A \in \mathrm{SL}\left(2, \mathbb{Z}_{p}\right): \operatorname{tr} A=0\right)=p(p-1),
$$

в то время как при $p=4 c+1$ особые значения дают вклад $2(2 p-1)$, так что

$$
\# A=(p-2)(p-1)+2(2 p-1)=\left(p^{2}-3 p+2\right)+(4 p-2)=p(p+1) \text {. }
$$

С другой стороны, элементы $\left(\begin{array}{ll}\alpha & \beta \\ \gamma & \delta\end{array}\right)$ стационарной группы $H$ стандартной структуры $I$ определяются уравнением

$$
\left(\begin{array}{ll}
\alpha & \beta \\
\gamma & \delta
\end{array}\right)\left(\begin{array}{cc}
0 & 1 \\
-1 & 0
\end{array}\right)=\left(\begin{array}{cc}
0 & 1 \\
-1 & 0
\end{array}\right)\left(\begin{array}{ll}
\alpha & \beta \\
\gamma & \delta
\end{array}\right), \quad\left(\begin{array}{cc}
-\beta & \alpha \\
-\delta & \gamma
\end{array}\right)=\left(\begin{array}{cc}
\gamma & \delta \\
-\alpha & -\beta
\end{array}\right)
$$


$\alpha=\delta, \gamma=-\beta$, с условием унимодулярности $\alpha^{2}+\beta^{2}=1$. По лемме $1 \S 2$ число элементов этой (круговой) группы $H$ равно $p \pm 1$ при $p=4 c \mp 1$. Следовательно, число комплексных структур, сопряженных со стандартной, есть $|G| /|H|=p\left(p^{2}-1\right) /(p \pm 1)=p(p \mp 1)$ при $p=4 c \mp 1$.

Это число совпадает с числом унимодулярных матриц со следом 0 в $G$.

Значит, все матрицы из $G$ со следом нуль сопряжены в группе $G$ стандартной структуре I, что и доказывает лемму 4.

Вернемся к окончанию доказательства леммы 3. Мы уже знаем, что цепочка квадратов $\left\{A_{s}\right\}$ заканчивается матрицами $A_{r-1}=-1$ и $A_{r}=1$. Если $r \geqslant 2$, то им предшествует комплексная структура $A_{r-2}$, сопряженная по лемме 4 стандартной структуре $I: A_{r-2}=g I g^{-1}$. Сопрягая всю цепочку квадратов $\left\{A_{s}\right\}$ тем же элементом $g$ группы $G$, мы получим новую цепочку квадратов, $\widetilde{A}_{s}=g A_{s} g^{-1}$, заканчивающуюся тройкой $\widetilde{A}_{r-2}=I, \widetilde{A}_{r-1}=I^{2}=-1, \widetilde{A}_{r}=1$.

Заметим, что след ни одной из матриц $\widetilde{A}_{s}$ (или $A_{s}$ ) с $s<r-2$ нулю не равен, так как иначе мы получили бы $A_{s}^{2}=-1, A_{s+2}=1$ при $s+2<r$, т. е. исходная цепочка имела бы меньшую $r$ длину.

Формула $(*)$ при $b t+c t=0, t \neq 0$, показывает, что $b+c=0$. Поэтому все матрицы полученной цепочки квадратов $\widetilde{A}_{s}$ при $s \leqslant r-2$ имеют $b+c=0$.

Точно так же формула $(*)$ при $(a t-1)-(d t-1)=0, t \neq 0$, показывает, что $a=d$. Значит, все матрицы цепочки квадратов $\left\{\widetilde{A}_{s}\right\}$ при $s \leqslant r-2$ принадлежат круговой группе $C_{p}=\left\{\left(\begin{array}{cc}a & b \\ -b & a\end{array}\right)\right\}$. Лемма 3 доказана. Причем, согласно теореме о цикличности из $\S 2$, в случаях $p=2^{r} c \pm 1$ мы получаем в $C_{p}$ цепочку квадратов длины $r\left(A_{r}=1\right)$.

Теорема 3 вытекает из этой леммы, так как порядок круговой группы $C_{p}$ равен $p \pm 1$ (при $p=4 c \mp 1$ ) по теореме 2, а порядок построенной выше ее циклической подгруппы $\left\{\widetilde{A}_{0}^{k}\right\}$ равен $2^{r}$. Значит, число $p \pm 1$ делится на $2^{r}$. В зависимости от остатка при делении $p$ на 4 , мы приходим к выводам

$$
\begin{array}{lll}
p+1=2^{r} q & \text { при } p=4 c-1 & \text { (и тогда } \left.p=2^{r} q-1\right), \\
p-1=2^{r} q & \text { при } p=4 c+1 & \text { (и тогда } \left.p=2^{r} q+1\right) .
\end{array}
$$

И обратно, для таких значений $p$ цепочка квадратов длины $r$ предъявлена выше.

Тем самым теорема 3 доказана полностью.

\section{§4. Уравнение $A^{p}=1$ в группе $G=\mathrm{SL}\left(2, \mathbb{Z}_{p}\right)$ и геометрия Лобачевского}

Из матричной теоремы Ферма 11 мы получаем по модулю $p$ сравнение для $A$ из $G$

$$
\operatorname{tr}\left(A^{p}\right) \equiv(\operatorname{tr} A)^{p}=t^{p}, \quad \text { где } t=\operatorname{tr} A .
$$

Из обычной теоремы Ферма следует числовое сравнение $t^{p} \equiv t(\bmod p)$. Мы получаем матричную теорему Ферма в виде сравнения по модулю $p$

$$
\operatorname{tr}\left(A^{p}\right) \equiv \operatorname{tr} A .
$$

Для решения уравнения $A^{p}=1$ в $G$ начнем поэтому с исследования матриц $A$ со следом $\operatorname{tr} A=\operatorname{tr} 1=2$. 
Лемма 1. Матрищи со следом 2 образуют в группе $G=\mathrm{SL}\left(2, \mathbb{Z}_{p}\right)$ подмножество $M$ из $p^{2}$ элементов, замкнутое относительно операции возведения матрии в квадрат.

Доказательство. По формуле $(*)$ из $\S 3 \operatorname{tr}\left(A^{2}\right)=(\operatorname{tr} A)^{2}-2=4-2=2$, если $\operatorname{tr} A=2$. Чтобы сосчитать число элементов множества $M$, запишем эти матрицы в виде $A=1+B, \operatorname{tr} B=0, B=\left(\begin{array}{cc}x & y \\ z & -x\end{array}\right)$. Условие унимодулярности $\operatorname{det} A=1$ принимает вид $1-x^{2}-y z=1$, т.е. $y z=-x^{2}$. Если $x \neq 0$, то получаем $p-1$ упорядоченных разложений $y z=u$ числа $u=-x^{2}$. Если $x=0$, то число разложений равно $2 p-1$. Итого число элементов множества $M$ равно сумме произведений чисел возможных выборов числа $x$ и чисел разложений, $(p-1)^{2}+(2 p-1)=p^{2}$. Разумеется, в $M$ входят матрица 1 и жорданова матрица $J=\left(\begin{array}{ll}1 & 1 \\ 0 & 1\end{array}\right)$ (по-
рядка $p)$.

Собственные числа матрицы из $M$ определяются из характеристического уравнения $\lambda^{2}-2 \lambda+1=0$ и потому оба равны 1 для любой матрицъь из $M$. И обратно, любая матрица с двукратным собственным числом 1 принадлежит $M$.

Теорема 4. Множество $M$ матрии со следом 2 в $G=\mathrm{SL}\left(2, \mathbb{Z}_{p}\right)$ с нечетным простым р состоит из трех классов сопряэенных эементов: $1, M^{+} u$ $M^{-}$, где $\left(p^{2}-1\right) / 2$ элементов класса $M^{+}$сопряжсены жсордановой матрице $J$,

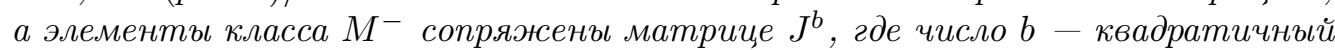
невычет по модулю $р$.

В этом смысле $p^{2}-1$ элементов множества $M \backslash 1$ решений уравнения $A^{p}=1$, $A \neq 1$, разбиваются на два равномощных класса эквивалентности соответственно разбиению неравных нулю вычетов по модулю $p$ на квадратичные и неквадратичные.

ДокАзАтельство. Стационарная группа $H$ жордановой матрицы $J$ состоит из унимодулярных матриц $\left(\begin{array}{ll}\alpha & \beta \\ \gamma & \delta\end{array}\right)$, для которых

$$
\left(\begin{array}{ll}
\alpha & \beta \\
\gamma & \delta
\end{array}\right)\left(\begin{array}{ll}
1 & 1 \\
0 & 1
\end{array}\right)=\left(\begin{array}{ll}
1 & 1 \\
0 & 1
\end{array}\right)\left(\begin{array}{ll}
\alpha & \beta \\
\gamma & \delta
\end{array}\right), \quad\left(\begin{array}{cc}
\alpha & \alpha+\beta \\
\gamma & \gamma+\delta
\end{array}\right)=\left(\begin{array}{cc}
\alpha+\gamma & \beta+\delta \\
\gamma & \delta
\end{array}\right),
$$

т. е. $\gamma=0, \alpha=\delta$. Условие унимодулярности дает $\alpha^{2}=1$. Число решений, при нечетном $p$, составляет $2 p$ (так как $\left.\alpha= \pm 1, \beta \in \mathbb{Z}_{p}\right):|H|=2 p$.

Поэтому число элементов, сопряженных в $G$ с $J$, равно $|G| /|H|=$ $p\left(p^{2}-1\right) /(2 p)=\left(p^{2}-1\right) / 2$.

В частности, матрица $J^{b}$ сопряжена матрице $J$, если и только если $b \neq 0$ - квадратичный вычет. Сопряженность всех отличных от 1 матриц следа 2 либо матрице $J$, либо матрице $J^{b}$ проверяется прямой выкладкой, аналогичной приведенной выше. Теорема 4 доказана.

ЗАмечание. С матрицей $A \neq 1$ из $M$ можно естественно связать ее единственную инвариантную прямую, $\pi A$, проходящую через 0 на конечной плоскости $\mathbb{Z}_{p}^{2}$, т. е. точку конечной проективной прямой $P=P^{1}\left(\mathbb{Z}_{p}\right)$. Обратно, по такой точке $\pi A \in P$ матрица $A$ из $M \backslash 1$ восстанавливается со следующей точностью: прообраз при отображении $\pi$ каждой из $p+1$ точек прямой $P$ среди 
$\left(p^{2}-1\right) / 2$ точек множества $M^{+}$состоит из $(p-1) / 2$ матриц с общим собственным вектором (например, вместе с матрицей $J$ в него входят матрицы $J^{b}$, для которых $b$ - квадратичный вычет по модулю $p$ ).

Каждый элемент $g$ группы $G$ действует на $p+1$ точек множества $P$, переставляя их некоторой специальной - проективной - четной перестановкой. Это проективное действие специальными четными перестановками $g_{*}$ точек конечной проективной прямой $P$ над $\mathbb{Z}_{p}$, состоящей из $p+1$ точек, гомоморфно действию группы $G$ на множестве матриц $M^{+}$сопряжениями (в том смысле, что $\left.\pi\left(g A g^{-1}\right)=g_{*}(\pi A)\right)$.

Если матрица $g$ принадлежит множеству $M^{+}$, то перестановка $g_{*}$ оставляет точку $\pi g$ проективной прямой $P$ на месте.

Все проективные преобразования конечной проективной прямой $P$, оставляющие на месте одну ее точку, образуют конечную группу $L$. Если выбрать координаты так, чтобы остающаяся на месте точка имела аффинную координату $u=\infty$ на $P^{1}$, то преобразования, образующие группу $L$, станут аффинными: $u \mapsto a u+b, a \neq 0$.

Поэтому число этих преобразований есть $|L|=p(p-1)$. Но поднимаются из проективной группы в группу $G=\operatorname{SL}\left(2, \mathbb{Z}_{p}\right)$ только те из них, для которых вычет $a$ квадратичен. Так что образом группы $G$ при описанном гомоморфизме в перестановки точек прямой $P$ является подгруппа $L^{+}$индекса 2 в $L,\left|L^{+}\right|=$ $p(p-1) / 2$, состоящая из преобразований вида $u \mapsto a u+b$, где $a=c^{2}, c \neq 0,-$ квадратичный вычет.

Конечная группа $L^{+}$является $(\bmod p)$ аналогом обычной плоскости Лобачевского. Эту плоскость можно ведь (см. [1]) определять как группу аффинных (и сохраняющих ориентацию) преобразований вещественной прямой: $\{u \mapsto a u+b\}$, где $a>0$, т. е. $a=c^{2}, c \neq 0$ (снабженной левоинвариантной римановой метрикой). Условие положительности, нужное в вещественном случае, заменяется в теории чисел квадратичностью вычета.

Можно надеяться, что указанная здесь связь уравнения $A^{p}=1$ в $G$ с конечной плоскостью Лобачевского (из $p(p-1) / 2$ точек) окажется полезной для анализа комбинаторики операции возведения матриц из $G$ в квадрат и соответствующих римановых поверхностей (описанных для $p=5$ и 7 в статье [2]) и их кубов Кеплера. Например, точкам и прямым плоскости Лобачевского и двойственного ей мира де Ситтера из [3] можно сопоставлять решения уравнений $A^{p}=1$ и $A^{3}=1$, о втором из которых сказано ниже.

Но идея заменять входящие в аналитические теории неравенства условиями квадратичности вычетов в теории чисел может иметь и другие приложения, кроме геометрии Лобачевского.

\section{§5. Уравнение $A^{3}=1$ в группе $G=\operatorname{SL}\left(2, \mathbb{Z}_{p}\right)$}

Будем решать это уравнение, записав его в виде $A^{2}=A^{-1}$, что приводит для матрицы $A=\left(\begin{array}{ll}a & b \\ c & d\end{array}\right)$ к системе уравнений

$$
a t-1=d, \quad b t=-b, \quad c t=-c, \quad d t-1=a
$$

(где $t=a+d-$ след), поскольку $A^{-1}=\left(\begin{array}{cc}d & -b \\ -c & a\end{array}\right)$. 
Мы заключаем, что $b(t+1)=c(t+1)=0$, поэтому либо $t=-1$, либо $b=c=0$.

В первом случае условие $A^{2}=A^{-1}$ выполнено. Во втором случае мы находим $(a+d) t-2=t, t^{2}-t-2=0,(t+1)(t-2)=0, t=2$. При $a+d=2, a d=1$ мы заключаем, что $a=d=1$, так что второй случай доставляет лишь матрицу $A=1$. Итак, доказана

Лемма 1. Oтличные от 1 решения уравнения $A^{3}=1$ в $G$ суть в точности матрици следа -1 и определителя 1 из $G$ :

$$
A=\left(\begin{array}{cc}
a & b \\
c & -1-a
\end{array}\right), \quad-b c=a^{2}+a+1
$$

ПРимеР. Матрица $F=\left(\begin{array}{cc}0 & 1 \\ -1 & -1\end{array}\right)$ имеет куб 1 при любом $p$.

Чтобы найти число всех таких матриц $A$, исследуем по отдельности случаи $u=0$ и $u \neq 0$, где $u=a^{2}+a+1$.

ЛЕмма 2. Число решений а уравнения $a^{2}+a+1=0$ в $\mathbb{Z}_{p}$ равно 2 при $p=3 c+1$ (например, при $p=7,13$ ) и равно 0 при $p=3 c-1$ (например, при $p=5,11)$; при $p=3$ решение одно, $a=1$.

ДокАЗАтЕльство. Если $a^{2}+a+1=0$, то $a^{3}=1$. Обозначая через $\rho$ первообразный по модулю $p$ вычет, мы получаем $a=\rho^{\lambda}, a^{3}=\rho^{3 \lambda}$, и показатель $\lambda$ должен удовлетворять условию

$$
3 \lambda=k(p-1), \quad k \in \mathbb{Z} .
$$

Если число $k$ кратно 3 , то $a=1$. Условие $a^{3}=1$ всегда выполнено для этого $a$, но этот корень - лишний, так как при $a=1$ число $a^{2}+a+1=3$ равно 0 только при $p=3$.

Если же целое число $k$ не делится на 3 , то $p-1=3 c, p=3 c+1$. Выбирая $k=1, \lambda=c$, мы находим один из корней $a_{1}$ квадратного уравнения для $a$ (второй корень есть $a_{2}=1 / a_{1}=-a_{1}-1$ ). Лемма 2 доказана.

Теорема 5. Все отличные от 1 решения уравнения $A^{3}=1$ в $G=\mathrm{SL}\left(2, \mathbb{Z}_{p}\right)$ сопряжень решению $F$, и их число равно, в зависимости от остатка при делении простого числа р на 3 ,

$$
p(p-1) \quad \text { nри } p=3 c-1, \quad \text { p }(p+1) \quad \text { nри } p=3 c+1 .
$$

Интересно, что эти числа, а возможно и решения, связаны с точками и прямыми на конечных плоскостях Лобачевского и на их накрытиях $L$, описанных выше.

ДокАЗАТЕЛЬСТво. Из леммы 2 находим число матриц следа -1 и определителя 1 в зависимости от остатка от деления числа $p$ на 3 :

$$
\begin{gathered}
p(p-1) \quad \text { при } p=3 c-1, \\
(p-2)(p-1)+2(2 p-1)=\left(p^{2}-3 p+2\right)+(4 p-2)=p(p+1) \quad \text { при } p=3 c+1 .
\end{gathered}
$$


Стационарную подгруппу $H$ матрицы $F$ в $G$ ищем из уравнений

$$
\begin{aligned}
\left(\begin{array}{ll}
\alpha & \beta \\
\gamma & \delta
\end{array}\right)\left(\begin{array}{cc}
0 & 1 \\
-1 & -1
\end{array}\right) & =\left(\begin{array}{cc}
0 & 1 \\
-1 & -1
\end{array}\right)\left(\begin{array}{ll}
\alpha & \beta \\
\gamma & \delta
\end{array}\right), \\
\left(\begin{array}{ll}
-\beta & \alpha-\beta \\
-\delta & \gamma-\delta
\end{array}\right) & =\left(\begin{array}{cc}
\gamma & \delta \\
-\alpha-\gamma & -\beta-\delta
\end{array}\right),
\end{aligned}
$$

т. е. $\gamma=-\beta, \delta=\alpha-\beta$. Из условия унимодулярности $\operatorname{det} A=1$ получаем рациональную кривую $\alpha^{2}-\alpha \beta+\beta^{2}=1$. Полагая $\alpha=x-1, \beta=t x$, находим для второй точки пересечения этой кривой с прямой $\beta=t x$, проходящей через точку $(\alpha=-1, \beta=0)$ нашей кривой, уравнение

$$
\begin{gathered}
x^{2}\left(1-t+t^{2}\right)+x(t-2)=0, \\
x=\frac{2-t}{1-t+t^{2}}, \quad \alpha=\frac{1-t^{2}}{1-t+t^{2}}, \quad \beta=\frac{2 t-t^{2}}{1-t+t^{2}} .
\end{gathered}
$$

Чтобы исключить бесконечно удаленные (в направлениях асимптот) точки из числа $p+1$ точек, получаемых при $t=1,2, \ldots, p, \infty$, решаем квадратное уравнение $1-t+t^{2}=0$ (из которого следует $t^{3}=-1$, что исследовать легче).

Если $t=\rho^{\lambda}$ для первообразного по модулю $p$ вычета $\rho$, то показатель $\lambda$ должен удовлетворять сравнению

$$
3 \lambda \equiv(p-1) / 2 \quad(\bmod (p-1)), \quad 6 \lambda=(p-1)(2 k+1) .
$$

Если целое число $2 k+1$ делится на 3 , то $t= \pm 1$. В этих случаях $1-t+t^{2}=0$ только для $p=3, t=+1$.

Если целое число $2 k+1$ не делится на 3 , то $p-1=3 c, p=3 c+1$. В этом случае наша формула для $\lambda$ доставляет (два) решения $t$ нашего квадратного уравнения в $\mathbb{Z}_{p}$, тогда как при $p=3 c-1$ их нет.

Окончательно мы получаем для порядка стационарной группы величину, зависящую от остатка при делении $p$ на 3 :

$$
|H|= \begin{cases}p+1 & \text { при } p=3 c-1, \\ p-1 & \text { при } p=3 c+1 .\end{cases}
$$

Следовательно, число сопряженных матрице $F$ элементов группы $G$ равно $|G| /|H|=p(p \mp 1)$ при $p=3 c \mp 1$, как и число матриц следа -1 в $G$. Значит, все эти матрицы сопряжены матрице $F$, и теорема 5 доказана (при $p \neq 3$ ).

При $p=3$ число матриц с $A^{3}=1$ в $G=\mathrm{SL}\left(2, \mathbb{Z}_{3}\right)$ равно 8 (это все матрицы следа -1$)$, в том числе 4 сопряжены матрице $F$, а 4 - матрице $F^{2}$.

Все это видно, например, из строения графа монады возведения в квадрат для группы $G$, имеющего вид $T_{1,1,6}+8 A_{2}$ (в обозначениях статьи $[2]$ ).

\section{ЛиТЕРАТУРА}

1. Арнольд В. И., Авеи, А. Эргодические проблемы классической механики. Ижевск, 1999, приложение 20, с. 168-176 (Arnold V., Avez A. Problèmes Ergodiques de la Mécanique Classique. Paris, Gautier-Villars, 1967.)

2. Арнолъд В. И. Топология и статистика формул арифметики. УМН, 58, вып. 4 (352), 3-28 (2003). 
3. Arnold V. I. Arithmetics of binary quadratic forms, symmetry of their continued fractions and geometry of their de Sitter world. Bull. Braz. Math. Soc., N.S., 34, No. 1, 1-41 (2003); MCCME, Dubna, 2002.

4. Girard A. Invention nouvelle en l'algèbre. Amsterdam, 1629.

5. Newton I. Arithmetica universalis. Cambridge, 1707, pp. 57-63.

Математический институт им. В. А. Стеклова,

Поступило в редакцию

CEREMADE, Universitè Paris-Dauphine

3 октября 2003 г. 